\title{
Blind Separation of Radar Signals Based on Time- Frequency Analysis of Short Time Fourier Transformation
}

\author{
CHENG Xu-De, XUE Xue-Dong, XU Bing, ZHENG Yuan, WANG Pin \\ Wu Han Mechanical Technology College, Wuhan Hubei 430075, China \\ xxdmymail1228@126.com
}

\begin{abstract}
Radar signal sorting is a key part of radar countermeasure nowadays, a blind separation algorithm based on Time-Frequency (TF) analysis of short time Fourier transformation is proposed in this paper, and the problems of underdetermined radar signal sorting can be solved effectively. The proposed method avoids some of the drawbacks in literature, relax the assumption by allowing the sources to be TFnondisjoint to a certain extent, it can be achieved the algorithm in the condition of TF-nondisjoint. And this method has been successfully applied to radar signal sorting process, the time domain waves of radar signals can be got and the whole radar signal sorting process is accomplished. In the simulation experiments, the performance and applications in different SNR condition of proposed method are discussed, simulation results verify the feasibility and superiority.
\end{abstract}

Keywords-Time-Frequency Analysis; Short Time Fourier Transformation (STFT); Radar Signal; Blind Separation; Subspace Projection

\section{INTRODUCTION}

Blind separation of radar signals is an important part of radar countermeasures. The purpose of blind separation of signals is to separate signal in each source, and "blind" sorting is mainly embodied by ${ }^{[5]-[6]}$ : 1 . The way in which source signals are mixed in the space is unknown; 2 . Due to antagonistic relationship between radar countermeasures, priori knowledge on radar source signal is zero to radar reconnaissance side. Moreover, with booming modern radar technology, radar netting as a mature technology has been applied to modern battlefield, which will, in most case, come into a situation of underdetermined condition as a result of the number of radar source signals more than that of receiving antenna. Therefore, it's necessary to find a kind of algorithm for effective blind separation of radar signals under underdetermined condition.

Blind source separation technology was evolved rapidly in recent years. It has been applied to many fields, such as communication, speech signal processing ${ }^{[9]-[10]}$, bioengineering $^{[4]}$, radar signal processing and etc, and corresponding algorithms are introduced for settlement of much puzzles. To deal with the problem of Underdetermined Blind Source Separation (UBSS), many experts and scholars have proposed corresponding solutions. In literature [1], the author introduced blind source separation algorithm based on STFT, which solved the problem of blind source separation of sparse speech signals under underdetermine condition, however, it's limited by the assumed condition of TFnondisjoint projection of source signals, which, if not be met, will invalidate the algorithm any more. Also in literatures [2][3], the algorithm of blind source separation under underdetermine condition is introduced, mainly by means of two-order TF analysis and linear TF analysis, likewise, these two methods must also be applied with the assumption of TFnondisjoint or quasi TF-nondisjoint projection of source signals.

\section{DESCRIPTION OF PROBLEM}

\section{A. Mathematic Model}

Assume $N$ radar source signals need to be separated, expressed by $s_{1}(t), s_{2}(t), \ldots, s_{N}(t) ; M$ observed signals after mixing are intercepted by $M$ receiving antenna, expressed by $x_{1}(t), x_{2}(t), \ldots, x_{M}(t)$; then, intercepted signals and source signals can be expressed by ${ }^{[7]}$ :

$$
x(t)=A s(t)
$$

Where: $s(t)=\left[s_{1}(t), s_{2}(t), \ldots, s_{N}(t)\right]^{T}$, is signals matrix from transposition and combination of source signal vectors; "T" means transposition; $x(t)=\left[x_{1}(t), x_{2}(t), \ldots, x_{M}(t)\right]^{T}$, is matrix of intercepted signals by multiplying matrix of source signals and mixing matrix; to make underdetermined condition,

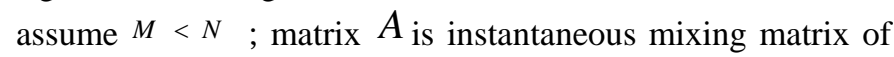
$M \times N, A=\left[\alpha_{1}, \alpha_{2} \ldots, \alpha_{N}\right]^{T}$; here, $A$ of mixing matrix is constrained:

In matrix $A$, no multiple relation exists among column vectors; if in the mixing matrix, $\alpha_{i}=k \alpha_{j}$, and $i, j \in N$, $i \neq j$, it'll lead to failure of separation of $s_{i}(t), s_{j}(t)$ in source signals.

Without loss of generality of the algorithm, by the uncertainty of mixing matrix, unitize all column vectors of mixing matrix, i.e. $\|\alpha\|=1,, \quad i \in 1, \ldots, N$.

\section{B. Time-Frequency Analysis}

TF analysis provides a favorable platform for solving the problem of non-stationary processing. TF analysis is the extension of time domain analysis and frequency domain analysis, which distributes the energy of processed signals in time domain and frequency domain, also called TimeFrequency Distribution (TFD). 


$$
\begin{aligned}
\zeta_{z z}(t, f) & \triangleq \iiint_{-\infty}^{\infty} e^{j 2 \pi v(u-t)} \Gamma(v, \tau) \\
& \times z\left(u+\frac{\tau}{2}\right) z^{*}\left(u-\frac{\tau}{2}\right) e^{j 2 \pi f \tau} d v d u d \tau
\end{aligned}
$$

Formula (2) is the basic method for deriving two-order TFD, where $\Gamma(v, \tau)$ means two-dimensional function for the control of the characters of signal TFD. In this paper, TF analysis of signals is conducted based on STFT.

$$
S_{z}(t, f) \triangleq \int_{-\infty}^{\infty} z(\tau) h(\tau-t) e^{-j 2 \pi f \tau} d \tau
$$

Formula (2) is definition formula of STFT, where: $z(\tau)$ is source signal analyzed; $h(\tau-t)$ is window function $S_{z}(t, f)$ is TFD of $\operatorname{signal}^{z(\tau)}$; the spectrum of signal $z(\tau)$ is defined as follows:

$$
\zeta_{z z}^{S P E C}(t, f) \triangleq\left|S_{z}(t, f)\right|^{2}
$$

It's obvious from definition formula of STFT that no cross term will be generated from STFT processing of signal, in comparison with another common TF analysis method Wigner-Ville Distribution (WVD).

$$
\zeta_{z z}^{W V D}(t, f) \triangleq \int_{-\infty}^{\infty} z\left(t+\frac{\tau}{2}\right) z^{*}\left(t-\frac{\tau}{2}\right) e^{-j 2 \pi f \tau} d \tau
$$

TFD resolution derived from STFT processing is lower than WVD resolution, but WVD is affected by cross term, thus the merits of TFD and WVD are combined and defined as Masked Wigner-Ville Distribution (MWVD) as TFD spectrum diagram of source signals.

$$
\zeta_{z z}^{M W V D}(t, f) \triangleq \zeta_{z z}^{W V D}(t, f) \cdot \zeta_{z z}^{S P E C}(t, f)
$$

Formula (5) indicates that MWVD is featured by high resolution of WVD and multiplication of the spectrum from STFT processing, in this way, the interference of cross term is eliminated.

\section{INTRODUCTION OF ALGORITHM}

Assume source signals of $z(t)=\left[z_{1}(t), \ldots, z_{N}(t)\right]^{T}$, and it's derived from Formula (2) that:

$$
D_{z z}(t, f) \triangleq\left[\begin{array}{ccc}
\zeta_{z_{1} z_{1}}(t, f) & \ldots & \zeta_{z_{1} z_{N}}(t, f) \\
\ldots & \ldots & \ldots \\
\zeta_{z_{N} z_{1}}(t, f) & \ldots & \zeta_{z_{N} z_{N}}(t, f)
\end{array}\right]
$$

Where: $\zeta_{z_{i} z_{j}}(t, f)$ means TFD between two signal vectors, $D_{z_{i} z_{j}}(t, f)$

means cross TFD matrix, it's derived from STFT processing of two sides of Formula (1), that:

$$
D_{x x}(t, f)=A D_{s s}(t, f) A^{H}
$$

Where: $S_{x x}(t, f)$ means TFD of observed signals; $S_{s s}(t, f)$ means TFD of source signals; energy distribution of signals is reflected on TFD diagram of the signals; defined in TFD: energy gain point generated from source signals is called automatic gain TF point; energy gain point generated from cross term is called cross gain point; energy distribution at other points is zero.

Set up time-frequency condition as described in 1.3; assume the set $\Omega$ of all points of source signals in TFD, of which one automatic gain point is $\left(t_{a}, f_{a}\right) \in \Omega$, then Formula (8) can be simplified for this automatic gain point, as follows:

$$
S_{x}\left(t_{a}, f_{a}\right)=\tilde{A} S_{\tilde{s}}\left(t_{a}, f_{a}\right)
$$

Where: $\tilde{S}$ is matrix of all signals at automatic gain point $\left(t_{a}, f_{a}\right)$; assume the number of signals at one automatic gain point is $\alpha_{k}$, expressed by $\left[s_{\alpha_{1}}, \ldots, s_{\alpha_{k}}\right]^{T} ; \tilde{A}$ is mixing matrix corresponding to $\tilde{S}$, expressed by $\left[\alpha_{\alpha_{1}}, \ldots, \alpha_{\alpha_{k}}\right]$; in essence, the problem of blind source separation is un-mixing of mixed signals at one automatic gain point. Assume $Q$ matrix is normal projection of $\tilde{A}$ on noise subspace, then:

$$
Q=I-\tilde{A}\left(\tilde{A}^{H} \tilde{A}\right)^{-1} \tilde{A}^{H}
$$

It's obvious from Formula (10) that, only the matrix of signal vectors at automatic gain point enables $Q$ matrix to zero, that is:

$$
\left\{\begin{array}{l}
Q=0, \alpha_{i} \in \tilde{A} \\
Q \neq 0, \alpha_{i} \notin \tilde{A}
\end{array}\right.
$$

Formula (11) gave the basis by which whether the vector belongs to signal matrix is judged and estimated; assume component vectors of mixing matrix $A$ are estimated by a given approach, corresponding signal vector can be judged from Formula (11); as long as estimated mixing matrix $A$ is derived, source signal can be derived.

$$
\hat{S}_{\tilde{S}}(t, f) \approx \tilde{A}^{+} S_{x}(t, f)
$$

Where: "+” is generalized inverse matrix; then, mixing matrix $A$ should be estimated; particular method for estimation of mixing matrix $A$ is specified hereinafter; at first, directional vectors of the space at automatic gain point are derived:

$$
v\left(t_{a}, f_{a}\right)=\frac{S_{x}\left(t_{a}, f_{a}\right)}{\left\|S_{x}\left(t_{a}, f_{a}\right)\right\|}
$$

Then, these vectors are clustered by $k$ K-means clustering, and finally clustered to classification ${ }^{N}=\left\{C_{i} \mid i \in 1, \ldots, N\right\}$; in classification ${ }^{C_{i}}$, remove estimated directional vector that is farthest from classification center, so as to reduce the range of clustering, and increase the precision of estimation; then, classify all directional vectors at automatic gain point, and finally estimate various vectors of mixing matrix, as follows:

$$
\hat{a}_{i}=\frac{1}{\left|C_{i}\right|} \sum_{(t, f) \in \Omega} v(t, f)
$$




\section{SIMULATION EXPERIMENT}

For this simulation, 4 radar source signals and 3 receiving antenna are set to form underdetermined condition; all radar signals are Linear Frequency Modulated (LFM) mutually overlapped on TF plane and formed time-frequency condition of signals; $\mathrm{SNR}=10 \mathrm{~dB}$; simulation results are as follows:

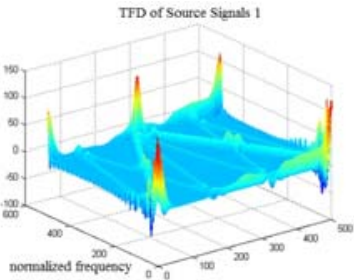

(1)

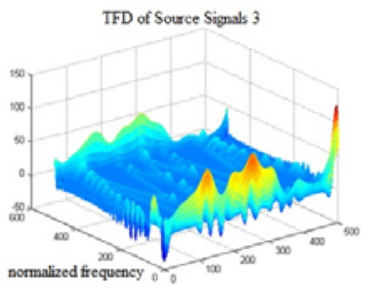

(3)

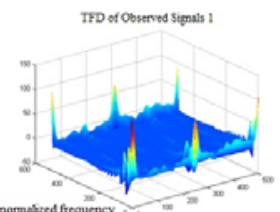

(5)

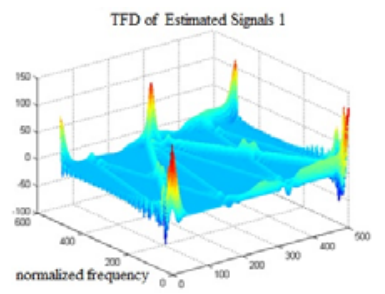

(8)

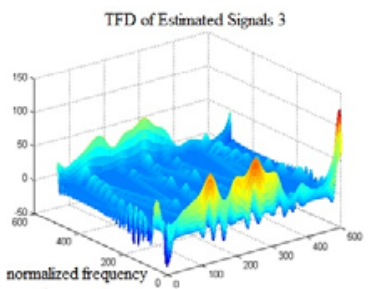

(10)

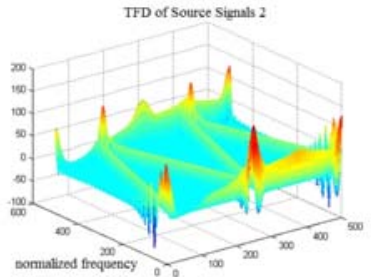

(2)

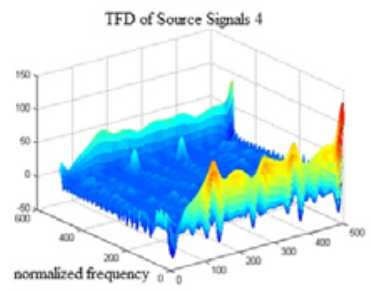

(4)

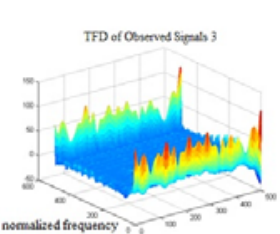

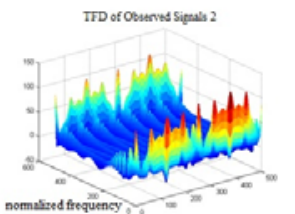

(6)
(7)

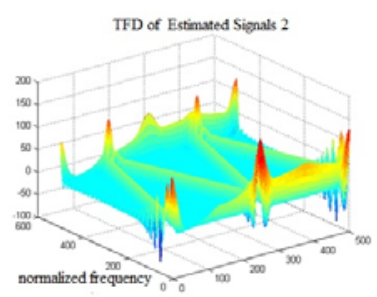

(9)

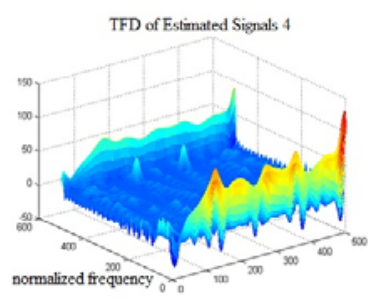

(11)
Fig. 1 TFD of Source Signals, Observed Signals and Estimated Signals in Radar Signal Sorting

In Fig.1, (1)-(4) are TFD of radar source signals; (5)-(7) are TFD of signals intercepted by receiving antenna; (8)-(11) are TFD of estimated radar source signals; time domain waveform of radar source signals and time domain waveform of estimated radar source signals are as shown in Fig. 2:

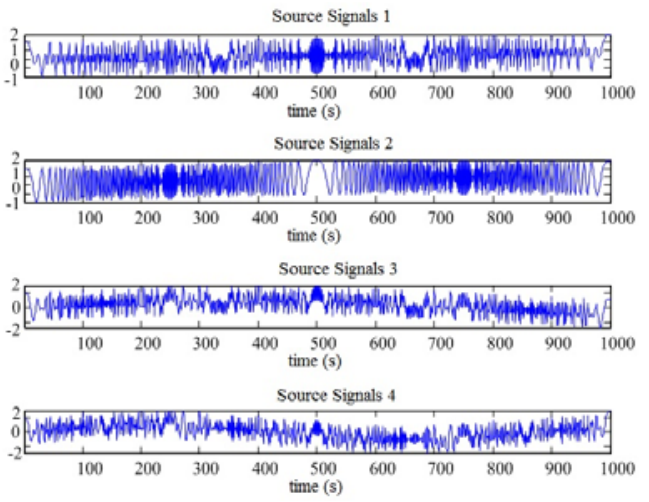

(1)

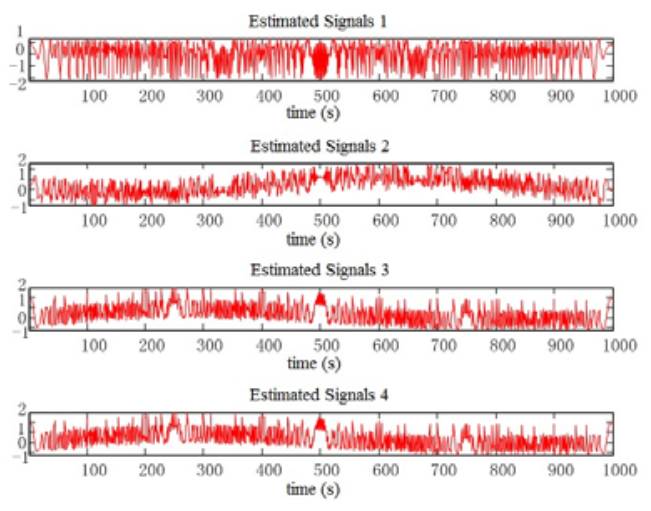

(2)

Fig.2 Time Domain Waveforms of Source Signals

Figures 2(1) (2) reflect time domain waveforms of source signals and estimated signals, respectively. It's apparently shown on Figure 1 and 2 that blind separation of radar signals is realizable with this algorithm under underdetermined condition. The particular results are analyzed as follows: first, define estimation error $E_{A}$, that is:

$$
E_{A}=10 \lg \left(\frac{1}{N}\|z-\hat{z}\|_{F}\right)
$$

Where: $\hat{Z}$ means estimated signal; \|\|$_{F}$ is $F$ norm; $N$ is number of experiments; then, the less estimated error, the higher estimation precision; the more estimated error, the lower estimation precision; in the experiment, sorting effect of this algorithm is measured by matrix estimation error $E_{A}$.

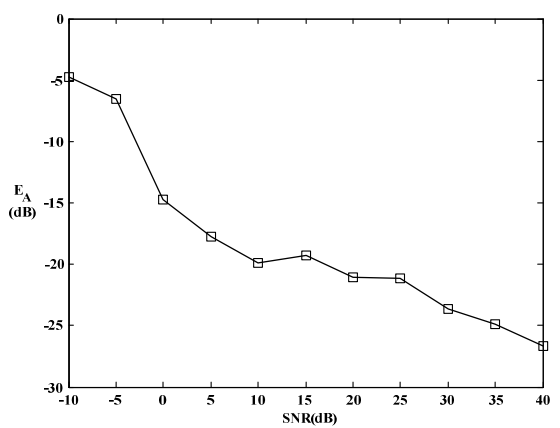

Fig. 3 Comparison of Estimated Signals and Time Domain Signals of Source Signals 
In the experiment, SNR increases from $-10 \mathrm{~dB}$ to $40 \mathrm{~dB}$, SNR step is $5 \mathrm{~dB}$; source signals are 4 LFM signals, 3 receiving antenna; angles of incidence sources signals are $15^{\circ}$, $30^{\circ}, 45^{\circ}$ and $60^{\circ}$, respectively; source signals intercepted by receiving antenna are separated by the algorithm introduced here; 100 cycles of Monte Carlo analysis are conducted under each SNR condition; finally, error of time domain waveform is derived from Formula (15) under various SNR conditions; Fig. 3 is error curve from the experiment; the higher SNR corresponds to lower estimation error, which conforms to theory; when $\mathrm{SNR}=-10 \mathrm{~dB}$, estimation error $=-5 \mathrm{~dB}$ is the maximum error in the experiment; this curve proves the workability of the algorithm introduced above. To validate other feature of this approach, following experiments are conducted:

Experiment 1: validate sorting performance of the algorithm under the condition of different numbers of receiving antenna.

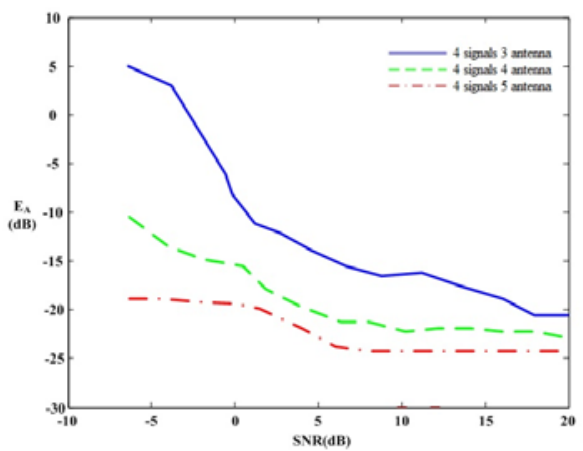

Fig. 4 Estimation Error of the Algorithm under the Condition of Different Number of Receiving Antenna

In experiment 1 , the condition of source signals as input remained unchanged, SNR increases from $-5 \mathrm{~dB}$ to $20 \mathrm{~dB}$ at a step of 5dB; 00 cycles of Monte Carlo analysis are conducted under each SNR condition; change the number of receiving antenna, as shown in Fig. 4, the lowest sorting precision is gained under the condition of 4 source signals and 3 receiving antenna; sorting precision is the highest under the condition of 4 source signals and 5 receiving antenna, which also reflects that relation between the number of signals and number of antenna is a major factor having impact on the precision of sorting algorithm.

Experiment 2: comparison with high order statistics (HOS)

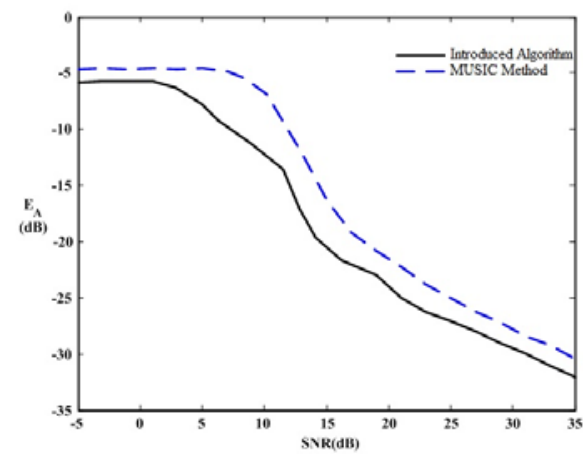

Fig. 5 Comparison of Introduced Algorithm with MUSIC Method
Experiment 2 is conducted under underdetermined condition; SNR increases from $-5 \mathrm{~dB}$ to $35 \mathrm{~dB}$ at a step of $5 \mathrm{~dB}$, the purpose is to explore the estimation precision with MUSIC method and introduced algorithm. As shown in Fig. 5, due to increase in SNR, estimation error is reduced with both methods; however, it's obvious that estimation error with signal sorting algorithm based on the analysis of short time Fourier time-frequency transformation introduced in this paper is always less than that with currently conventional high order statistics MUSIC method, indicating that our introduced algorithm is better than MUSIC method.

\section{CONCLUSION}

A kind of algorithm for blind separation of radar signals under underdetermined condition is introduced in this paper. Particular steps of blind source separation are described, the effectiveness of this algorithm in solving the problem of radar signal sorting is validated through simulation experiments, and the introduced algorithm is compared with MUSIC method and indicated better estimation precision. Blind source separation has been applied to radar signal sorting module of airborne radar warning receiver simulating system, where favorable sorting performance is embodied with rewarding value in actual application.

\section{References}

[1] O. Yilmaz, S. Rickard. Blind separation of speech mixtures via timefrequency masking [J]. IEEE Trans. Signal Process. 2004, 52(7): 18301847.

[2] N. Linh-Trung, A. Belouchrani, K. Abed-Meraim, et al. Separating more sources than sensors using time-frequency distributions[J]. EURASIP J. Appl. Signal Process. 2005, 2005(17): 2828-2847.

[3] B. Barkat, K. Abed-Meraim. Algorithms for blind components separation and extraction from the time-frequency distribution of their mixture[J]. EURASIP J. Appl. Signal Process. 2004, 2004(13): 20252033.

[4] A. K. Nandi. Blind Estimation Using Higher Order Statistics[M]. Boston, MA: Kluwer Academic. 1999.

[5] SUN Hong, AN Huangbin. A Method to Select Radar Signal Based on Blind Source Separation [J]. Modern Radar. 2006, 23(3): 47-50.

[6] XIAO Wen-shu, ZHANG Xing-gan, DU Si-dan. Blind Separation of Radar Signals [J]. Journal of Nanjing University. 2006, 42(1): 38-43.

[7] CHEN Xiao-jun, CHENG Hao, TANG Bin. Underdetermined Blind Radar Signal Separation Based on ICA [J]. Journal of Electronics \& Information Technology. 2010, 32(4): 919-924.

[8] Ruan Zong-li, Li Li-ping, Qian Guo-bing, Luo Ming-gang. Fast Fixedpoint Algorithm Based on Complex ICA Signal Model with Noise [J]. Journal of Electronics \& Information Technology. 2014, 36(5): 10941099.

[9] Bin G, W. L. Woo, S. S. Dlay. Single-Channel Source Separation Using EMD-Subband Variable Regularized Sparse Features[J]. IEEE Transactions on Audio, Speech, and Language Processing. 2011, 19(4): 961-976.

[10] GU Fan, WANG Hui-gang, LI Hu-xiong. A Blind Speech Separation Algorithm with Strong Reverberation [J]. Signal Processing. 2011, 27(4): 534-540.

[11] Klaus N, Esa O, Hannu O. On the Performance Indices of ICA and Blind Source Separation[C]. 2011 IEEE 12th International Workshop on Signal Processing Advances in Wireless Communications. 2011: 461465. 